\title{
Е.А. Филатов
}

\section{ФАКТОРНЫЙ АНАЛИЗ МОДЕЛИ УСТОЙЧИВОСТИ ЭКОНОМИЧЕСКОГО РОСТА ПРЕДПРИЯТИЯ}

\author{
E.A. Filatov \\ FACTOR ANALYSIS OF THE MODEL INDICATING THE SUSTAINABLITY \\ OF THE ECONOMIC GROWTH OF THE ENTERPRISE
}

\begin{abstract}
Представлена ресурсно-управленческая модель степени устойчивости экономического роста, позволяющая более обоснованно выявлять первопричины изменения коэффициента устойчивости экономического роста. Анализ ресурсно-управленческой модели производится методом факторного интегрального анализа. Факторный анализ в современных условиях становится элементом управления, инструментом оценки надежности функционирования коммерческих организаций. Представленная ресурсноуправленческая модель степени устойчивости экономического роста предназначена для выявления и анализа факторов, определяющих эффективность функционирования коммерческих организаций, оценки степени их влияния и складывающейся тенденции в их изменении и значимости. Чтобы правильно оценить эффективность решений, принимаемых на основе данных анализа, управление предприятием должно быть обоснованным. Управленческие решения в современных условиях жесткой конкуренции не могут приниматься интуитивно, приблизительно, они должны быть основаны на точных расчетах, глубоком экономическом анализе. Данные результатов анализа являются, таким образом, базой для разработки и принятия управленческих решений. Экономический анализ является функцией управления, обеспечивающей научность принятия решений. Основой анализа является система показателей и аналитических таблиц, логика отбора и составления которых предполагает анализ состояния и динамики экономического потенциала предприятия, результатов и эффективности его использования. Факторный детерминированный анализ направлен на выявление влияния факторов на величину интересующего результативного показателя, исключающего погрешность, и он наиболее актуален для практического применения в условиях рыночных отношений. Представлен альтернативный подход по определению интегральной оценки влияния факторов на обобщающий показатель. Предложенный метод интегрального факторного анализа дает возможность наиболее доступно и менее трудоемко составить заключение об изменениях в финансовом положении предприятия, а также позволяет оценить степень влияния факторов на изменение исследуемого показателя в системе хозяйствования, а также тенденции его изменения.

ФАКТОРНЫЙ АНАЛИЗ; ИНТЕГРАЛЬНЫЙ МЕТОД; ОТКЛОНЕНИЕ; ДОПОЛНИТЕЛЬНЫЙ ПРИРОСТ РЕЗУЛЬТАТИВНОГО ПОКАЗАТЕЛЯ; ЭКОНОМИЧЕСКИЙ РОСТ.
\end{abstract}

The article presents the author's resource-management model indicating the sustainability of economic growth that allows identifying the primary causes of changes in the sustainability coefficient in a more substantiated way. The resource-management model is analyzed by applying the author's integral factor method. Recently, factor analysis has become an element of management, a tool to evaluate the operational safety of business entities. The introduced resource-management model of the sustainability of economic growth is designed to identify and analyze the factors determining the performance efficiency of commercial organizations, to evaluate their impact and emerging trends in their changes and significance. To properly evaluate the effectiveness of decisions taken on the basis of data analysis, enterprise management should be well reasoned. Under current highly competitive conditions, management decisions cannot be intuitive or approximate, they should be based on accurate calculations and thorough economic analysis. The results of data analysis thus constitute a basis for the development and execution of management decisions. Economic analysis is a management function that ensures the scientific character of decision-making. Such an analysis relies on the system of indicators and analytical tables, the logic of selection and preparation of which involves the analysis of the status and dynamics of the economic potential of the enterprise, the results and effectiveness of its use. Deterministic factor analysis aims to identify the influence of error-free factors on the value of the effective index. In the presence of market relations, it is most suitable for practical application. The article introduces an alternative approach to making an integral assessment of the impact of the factors on the generalizing indicator. The author's method of integral factor analysis gives an 
opportunity to make a more comprehensible and less time-consuming conclusion about changes in the financial state of a company as well as to evaluate the degree of the impact of the factors on the variations of the analyzed indicator in a management system and its change trends.

FACTOR ANALYSIS; INTEGRAL METHOD; DEFLECTION; ADDITIONAL GROWTH OF THE EFFECTIVENESS INDICATOR; ECONOMIC GROWTH.

Введение. Одной из наиболее важных проблем для современных коммерческих организаций является обеспечение своей экономической устойчивости в краткосрочной и долгосрочной перспективе. «Активный поиск методов и средств адаптации предприятий к трансформируемой экономической среде становится предпосылкой нейтрализации поразивших их деградационных процессов и воспроизводства ресурсов в конкурентном окружении» [1]. Эффективность используемых ресурсов зависит от качества управления организацией. Коэффициент устойчивости экономического роста характеризует темп роста собственного капитала за счет реинвестирования нераспределенной прибыли. Конкурентоспособность предприятию может обеспечить только правильное управление движением финансовых ресурсов и капитала, находящихся в распоряжении.

Повышение коэффициента устойчивости экономического роста организации возможно за счет повышения коэффициента реинвестирования, за счет улучшения уровня управления производством и реализации продукции, управления финансовыми, материальными, трудовыми ресурсами, улучшения использования основных фондов, повышения уровня финансовой независимости организации.

Финансовую устойчивость можно выразить и одним показателем, но он не будет характеризовать всю полноту взаимозависимости, которая характерна для нее. Комплексная оценка представляет собой характеристику, полученную в результате комплексного исследования, т. е. одновременного и согласованного изучения совокупности показателей, отражающих многие аспекты хозяйственных процессов и содержащих обобщающие выводы о результатах деятельности коммерческой организации.

Разработанная нами модель служит основой для оценки влияния различных факторов на динамику коэффициента устойчивости экономического роста, разработки объективных мероприятий по повышению финансового состояния. В модели, формула (1), связь исследуемых факторов с результативным показателем носит функциональный характер, т. е. результативный показатель факторной модели представлен в виде произведения факторов.

Коэффициент устойчивости экономического роста $\left(K_{e g}\right)$ - показатель, представляющий собой отношение реинвестированной прибыли к средней сумме собственного капитала. При этом исходная формула для проведения факторного анализа будет иметь следующий вид

$$
\begin{aligned}
K_{e g}= & \frac{Z K}{S K} \frac{S O K}{Z K} \frac{S A}{S O K} \frac{V}{S A} \frac{P P}{V} \frac{S S}{P P} \frac{P}{S S}= \\
= & F_{1} F_{2} F_{3} F_{4} F_{5} F_{6} F_{7} F_{8}=\prod_{n=1}^{8} F_{n},
\end{aligned}
$$

где $K_{e g}-$ коэффициент устойчивости экономического роста; $Z K-$ средняя стоимость заемного капитала; $S K$ - средняя стоимость собственного капитала; $S O K-$ средняя стоимость собственного оборотного капитала (собственные оборотные средства или рабочий капитал - сумма, на которую оборотные активы организации превышают ее краткосрочные обязательства); $S A$ - стоимость активов (капитала) - это сумма собственного и заемного капиталов; $V$ - выручка нетто, выручка от продажи продукции, товаров, работ, услуг, т. е. вся выручка, которую получает предприятие за вычетом налогов исчисляемых из нее (НДС, акцизов и аналогичных обязательных платежей); $P P$ - прибыль от продаж (это валовая прибыль за вычетом коммерческих и управленческих расходов); $S S$ - себестоимость продаж; $P$ - чистая прибыль (прибыль после уплаты налога на прибыль, прибыль подлежащая распределению); $R P$ - реинвестированная прибыль (прибыль, 
реинвестированная в расширение деятельности коммерческой организации, равняется чистой прибыли за вычетом дивидендов, начисленных в пользу акционеров); $F_{1}=Z K / S K-$ коэффициент финансового риска (плечо финансового рычага, коэффициент финансовой задолженности, коэффициент финансового риска); $F_{2}=S O K / Z K-$ коэффициент покрытия обязательств собственным оборотным капиталом (введен нами); $F_{3}=S A / S O K-$ коэффициент соотношения совокупного и собственного оборотного капитала (введен нами); $F_{4}=V / S A-$ коэффициент отдачи капитала (капиталоотдача, оборачиваемость капитала) показатель интенсивности использования организацией всей совокупности имеющихся активов; $F_{5}=P P / V-$ рентабельность продаж (расчет по прибыли от продаж); $F_{6}=S S / P P-$ коэффициент соотношения себестоимости и прибыли от продаж (введен нами); $F_{7}=P / S S-$ рентабельность производства (расчет по чистой прибыли); $F_{8}=R P / P-$ коэффициент реинвестирования (доля реинвестированной прибыли в общей сумме чистой прибыли).

Чтобы правильно оценить эффективность решений, принимаемых на основе данных анализа, управление предприятием должно быть обоснованным. Управленческие решения в современных условиях жесткой конкуренции не могут приниматься интуитивно, приблизительно, они должны быть основаны на точных расчетах, глубоком экономическом анализе. Данные результатов анализа являются, таким образом, базой для разработки и принятия управленческих решений. Экономический анализ является функцией управления, обеспечивающей научность принятия решений. Основой анализа является система показателей и аналитических таблиц, логика отбора и составления которых предполагает анализ состояния и динамики экономического потенциала предприятия, результатов и эффективности его использования.

«Основная идея экономического факторного анализа заключается в разложении общей вариации результирующей функции на отдельные, не зависящие друг от друга компоненты, каждый из которых характеризует влияние вариации того или иного фактора или взаимодействия целого ряда факторов» [2, c. 22].
Интегральный метод (прием дифференциально-интегральных исчислений) является приемом непоследовательного элиминирования. Данный метод ликвидирует основной недостаток, присущий традиционным приемам изучения прямой детерминированной факторной связи (цепных подстановок и метода разниц), - необходимость определения последовательности замены значений факторов, что иногда затруднительно при их большом числе, в то время как изменение последовательности влияет на результат расчета.

Интегральный метод (прием дифференциально-интегральных исчислений) позволяет достигнуть полного разложения результативного показателя по факторам и носит универсальный характер (разлагается дополнительный прирост результативного показателя в связи с взаимодействием факторов поровну между ними, независимо от их расположения в модели), т. е. данный способ применяется для измерения влияния факторов в мультипликативных, кратных и смешанных моделях кратно-аддитивного типа.

При использовании интегрального метода не учитывались особенности влияния количественных и качественных факторов; действия факторов считались равноценными, поэтому есть отклонения в результатах расчетов по факторам, по сравнению с расчетами, выполненными методом цепных подстановок и методом абсолютных и относительных разниц.

Традиционный интегральный метод для мультипликативных моделей применим только к двум и к трем факторам, входящим в функциональную модель [3-12]. Наш метод [13] можно использовать к любому количеству факторов, входящих в функциональную модель.

Основная задача предлагаемого интегрального метода факторного анализа, так же, как и известных, - выявить факторы, определяющие объем отраслевого предложения, т. е. суммарное изменение объема продукции со стороны основных факторов, его составляющих.

Условные исходные данные для проведения интегрального факторного анализа, представлены в табл. 1. 
Та блица 1

Исходные данные для проведения факторного интегрального анализа

\begin{tabular}{|c|c|c|c|}
\hline Показатели & План (0) & Факт (I) & Отклонение $(\Delta)$ \\
\hline$V$ - выручка нетто, тыс. руб. & 1000000 & 1200000 & 200000 \\
\hline$S S$ - себестоимость продаж, тыс. руб. & 820000 & 940000 & 120000 \\
\hline$Z K$ - средняя стоимость заемного капитала, тыс. руб. & 630000 & 700000 & 70000 \\
\hline$S K$ - средняя стоимость собственного капитала, тыс. руб. & 890000 & 1000000 & 110000 \\
\hline $\begin{array}{l}S A \text { - стоимость активов (капитала) или валюта баланса, } \\
\text { тыс. руб. }(3+4)\end{array}$ & 1520000 & 1700000 & 180000 \\
\hline $\begin{array}{l}S O K \text { - средняя стоимость собственного оборотного капитала, } \\
\text { тыс. руб. }\end{array}$ & 380000 & 400000 & 20000 \\
\hline PP - прибыль от продаж, тыс. руб. & 300000 & 380000 & 80000 \\
\hline$P$ - чистая прибыль, тыс. руб. & 280000 & 340000 & 60000 \\
\hline$R P$ - реинвестированная прибыль, тыс. руб. & 178000 & 300000 & 122000 \\
\hline $\begin{array}{c}K_{e g}-\text { коэффициент устойчивости экономического роста } \\
9 / 4=(11 \cdot 12 \cdot 13 \cdot 14 \cdot 15 \cdot 16 \cdot 17 \cdot 18)\end{array}$ & 0,2 & 0,3 & 0,1 \\
\hline$F_{1}-$ коэффициент финансового риска $(3: 4)$ & 0,70786517 & 0,7 & $-0,00786517$ \\
\hline $\begin{aligned} F_{2}- & \text { коэффициент покрытия обязательств } \\
& \text { оборотным капиталом }(6: 3)\end{aligned}$ & 0,6031746 & 0,57142857 & $-0,03174603$ \\
\hline $\begin{aligned} F_{3}- & \text { коэффициент соотношения совокупного и собствен- } \\
& \text { ного оборотного капитала }(5: 6)\end{aligned}$ & 4,0 & 4,25 & 0,25 \\
\hline$F_{4}-$ коэффициент отдачи капитала $(1: 5)$ & 0,65789474 & 0,70588235 & 0,047987616 \\
\hline$F_{5}-$ рентабельность продаж $(7: 1)$ & 0,3 & 0,31666667 & 0,016666667 \\
\hline $\begin{array}{c}F_{6}-\text { коэффициент соотношения себестоимости и прибыли } \\
\quad \text { от продаж }(2: 7)\end{array}$ & 2,73333333 & 2,47368421 & $-0,25964912$ \\
\hline$F_{7}-$ рентабельность производства $(8: 2)$ & 0,34146341 & 0,36170213 & 0,020238713 \\
\hline$F_{8}-$ коэффициент реинвестирования $(9: 8)$ & 0,63571429 & 0,88235294 & 0,246638655 \\
\hline
\end{tabular}

П р и м е ч а н и е . 0 - прошлый (базисный) период (год), взятый за базу сравнения; I - отчетный (текущий) период (год); $\Delta-$ изменение за период, рассчитывается как разница между фактом и планом (I - 0).

Совокупное отклонение по результатирующему показателю $\left(\Delta K_{e g}\right)$ определяется по формуле

$$
\begin{gathered}
\Delta K_{e g}=\sum_{n=1}^{8} \Delta K_{e g}\left(F_{n}\right)=\Delta K_{e g}\left(F_{1}\right)+\Delta K_{e g}\left(F_{2}\right)+ \\
+\Delta K_{e g}\left(F_{3}\right) \Delta K_{e g}\left(F_{4}\right)+\Delta K_{e g}\left(F_{5}\right)+\Delta K_{e g}\left(F_{6}\right)+(2) \\
+\Delta K_{e g}\left(F_{7}\right)+\Delta K_{e g}\left(F_{8}\right)
\end{gathered}
$$

Расчет влияния факторов на изменение результативного показателя представлен в следующих восьми формулах:

$$
\begin{aligned}
& \Delta K_{e g}\left(F_{1}\right)=\left(\Delta F_{1} / n\right)\left(F O_{1}\right)+Z, \\
& \Delta K_{e g}\left(F_{2}\right)=\left(\Delta F_{2} / n\right)\left(F O_{2}\right)+Z, \\
& \Delta K_{e g}\left(F_{3}\right)=\left(\Delta F_{3} / n\right)\left(F O_{3}\right)+Z, \\
& \Delta K_{e g}\left(F_{4}\right)=\left(\Delta F_{4} / n\right)\left(F O_{4}\right)+Z, \\
& \Delta K_{e g}\left(F_{5}\right)=\left(\Delta F_{5} / n\right)\left(F O_{5}\right)+Z, \\
& \Delta K_{e g}\left(F_{6}\right)=\left(\Delta F_{6} / n\right)\left(F O_{6}\right)+Z, \\
& \Delta K_{e g}\left(F_{7}\right)=\left(\Delta F_{7} / n\right)\left(F O_{7}\right)+Z, \\
& \Delta K_{e g}\left(F_{8}\right)=\left(\Delta F_{8} / n\right)\left(F O_{8}\right)+Z .
\end{aligned}
$$


Таблица 2

Выбор факторов для основной части формулы $\left(F O_{n}\right)$ по предложенному интегральному методу

\begin{tabular}{|c|c|c|c|c|c|c|c|c|c|c|c|c|c|c|}
\hline \multirow{3}{*}{$\begin{array}{c}\text { При влиянии } \\
\text { номера } \\
\text { фактора }\end{array}$} & \multicolumn{14}{|c|}{ Сумма сомножителей } \\
\hline & \multicolumn{7}{|c|}{ Сомножитель 1} & \multicolumn{7}{|c|}{ Сомножитель 2} \\
\hline & 0 & I & 0 & I & 0 & I & 0 & I & 0 & I & 0 & I & 0 & I \\
\hline 1 & 2 & 3 & 4 & 5 & 6 & 7 & 8 & 2 & 3 & 4 & 5 & 6 & 7 & 8 \\
\hline 2 & 1 & 3 & 4 & 5 & 6 & 7 & 8 & 1 & 3 & 4 & 5 & 6 & 7 & 8 \\
\hline 3 & 1 & 2 & 4 & 5 & 6 & 7 & 8 & 1 & 2 & 4 & 5 & 6 & 7 & 8 \\
\hline 4 & 1 & 2 & 3 & 5 & 6 & 7 & 8 & 1 & 2 & 3 & 5 & 6 & 7 & 8 \\
\hline 5 & 1 & 2 & 3 & 4 & 6 & 7 & 8 & 1 & 2 & 3 & 4 & 6 & 7 & 8 \\
\hline 6 & 1 & 2 & 3 & 4 & 5 & 7 & 8 & 1 & 2 & 3 & 4 & 5 & 7 & 8 \\
\hline 7 & 1 & 2 & 3 & 4 & 5 & 6 & 8 & 1 & 2 & 3 & 4 & 5 & 6 & 8 \\
\hline 8 & 1 & 2 & 3 & 4 & 5 & 6 & 7 & 1 & 2 & 3 & 4 & 5 & 6 & 7 \\
\hline
\end{tabular}

Дополнительный прирост результативного показателя в связи с взаимодействием факторов поровну между ними $(Z)$ представлен в формуле (4).

При использовании интегрального метода дополнительный прирост результативного показателя («неразложимый остаток» - Z), образовавшийся в результате взаимодействия факторов, распределяется между ними поровну.

$$
Z=\Delta K_{e g}-\Sigma\left(\left(\Delta F_{n} / n\right)\left(F O_{n}\right)\right) / n,
$$

где $Z$ - дополнительный прирост результативного показателя в связи с взаимодействием факторов поровну между ними; $F O_{n}-$ основная часть формулы авторского интегрального метода; $\Delta F_{n}-$ отклонение по определенному фактору; $n$ - количество факторов, участвующих в анализе.

Здесь $F O_{n}-$ основная часть формулы авторского интегрального метода, рассчитывается по восьми формулам:

$$
\begin{aligned}
& F O_{1}=2\left(\left(F_{2(0)} \cdot F_{3(I)} \cdot F_{4(0)} \cdot F_{5(I)} \cdot F_{6(0)} \cdot F_{7(I)} \cdot F_{8(0)}\right)+\right. \\
& \left.\quad+\left(F_{2(I)} \cdot F_{3(0)} \cdot F_{4(I)} \cdot F_{5(0)} \cdot F_{6(I)} \cdot F_{7(0)} \cdot F_{8(I)}\right)\right), \\
& F O_{2}=2\left(\left(F_{1(0)} \cdot F_{3(I)} \cdot F_{4(0)} \cdot F_{5(I} \cdot F_{6(0)} \cdot F_{7(I)} \cdot F_{8(0)}\right)+\right. \\
& \left.\quad+\left(F_{1(I)} \cdot F_{3(0)} \cdot F_{4(I)} \cdot F_{5(0)} \cdot F_{6(I)} \cdot F_{7(0)} \cdot F_{8(I)}\right)\right), \\
& F O_{3}=2\left(\left(F_{1(0)} \cdot F_{2(I)} \cdot F_{4(0)} \cdot F_{5(I)} \cdot F_{6(0)} \cdot F_{7(I)} \cdot F_{8(0)}\right)+\right. \\
& \left.\quad+\left(F_{1(I)} \cdot F_{2(0)} \cdot F_{4(I)} \cdot F_{5(0)} \cdot F_{6(I)} \cdot F_{7(0)} \cdot F_{8(I)}\right)\right), \\
& F O_{4}=2\left(\left(F_{1(0)} \cdot F_{2(I)} \cdot F_{3(0)} \cdot F_{5(I)} \cdot F_{6(0)} \cdot F_{7(I)} \cdot F_{8(0)}\right)+\right. \\
& \left.\quad+\left(F_{1(I)} \cdot F_{2(0)} \cdot F_{3(I)} \cdot F_{5(0)} \cdot F_{6(I)} \cdot F_{7(0)} \cdot F_{8(I)}\right)\right), \\
& F O_{5}=2\left(\left(F_{1(0)} \cdot F_{2(I)} \cdot F_{3(0)} \cdot F_{4(I)} \cdot F_{6(0)} \cdot F_{7(I)} \cdot F_{8(0)}\right)+\right. \\
& \left.\quad+\left(F_{1(I)} \cdot F_{2(0)} \cdot F_{3(I)} \cdot F_{4(0)} \cdot F_{6(I)} \cdot F_{7(0)} \cdot F_{8(I)}\right)\right), \\
& F O_{6}=2\left(\left(F_{1(0)} \cdot F_{2(I)} \cdot F_{3(0)} \cdot F_{4(I)} \cdot F_{5(0)} \cdot F_{7(I)} \cdot F_{8(0)}\right)+\right. \\
& \left.\quad+\left(F_{1(I)} \cdot F_{2(0)} \cdot F_{3(I)} \cdot F_{4(0)} \cdot F_{5(I)} \cdot F_{7(0)} \cdot F_{8(I)}\right)\right),
\end{aligned}
$$

$$
\begin{aligned}
& F O_{7}=2\left(\left(F_{1(0)} \cdot F_{2(I)} \cdot F_{3(0)} \cdot F_{4(I)} \cdot F_{5(0)} \cdot F_{6(I)} \cdot F_{8(0)}\right)+\right. \\
& \left.\quad+\left(F_{1(I)} \cdot F_{2(0)} \cdot F_{3(I)} \cdot F_{4(0)} \cdot F_{5(I)} \cdot F_{6(0)} \cdot F_{8(I)}\right)\right), \\
& F O_{8}=2\left(\left(F_{1(0)} \cdot F_{2(I)} \cdot F_{3(0)} \cdot F_{4(I)} \cdot F_{5(0)} \cdot F_{6(I)} \cdot F_{7(0)}\right)+\right. \\
& \left.\quad+\left(F_{1(I)} \cdot F_{2(0)} \cdot F_{3(I)} \cdot F_{4(0)} \cdot F_{5(I)} \cdot F_{6(0)} \cdot F_{7(I)}\right)\right) .
\end{aligned}
$$

Апробацию представленного метода факторного интегрального анализа см. в табл. 3, 4 .

Для формирования основной части формулы $\left(F O_{n}\right)$ необходимо воспользоваться принципом выбора факторов, раскрытых в табл. 2.

Количество показателей в основной части формулы $m$ определяется по формуле:

$$
m=n(2(n-1)) \text {. }
$$

При восьми факторах в модели $(n=8) m$ будет составлять 112: $(m=8(2 \cdot 7)=8 \cdot 14)$. К примеру: $n=9, m=144 ; n=10, m=180$; $n=11, m=220 ; n=12, m=264$ и т. д.

Как видим из итогового результата, цель предложенного интегрального метода достигнута - определение влияния факторов раскрыто без отклонений.

Bыводы. Одна из важнейших характеристик финансового состояния предприятия стабильность его деятельности с позиции долгосрочной перспективы.

Определение допустимых и экономически оправданных темпов развития коммерческих организаций - одна из главных задач финансового менеджмента, поэтому факторный анализ коэффициента устойчивости экономического роста выступает одним из самых важных инструментов, используемых при ее решении. 
Составные части формулы по предложенному интегральному методу

\begin{tabular}{|c|c|c|c|}
\hline \multirow{2}{*}{$\begin{array}{l}\text { Номер } \\
\text { формулы }\end{array}$} & \multicolumn{3}{|c|}{ Части формулы } \\
\hline & $\Delta F_{n} / n$ & $F O_{n}$ (основная часть) & $Z$ \\
\hline 1 & $\Delta K_{e g}\left(F_{1}\right)=\left(\Delta F_{1} / 8\right)$ & $\begin{array}{l}2\left(\left(F_{2(0)} \cdot F_{3(I)} \cdot F_{4(0)} \cdot F_{5(I)} \cdot F_{6(0)} \cdot F_{7(I)} \cdot F_{8(0)}\right)+\right. \\
\left.+\left(F_{2(I)} \cdot F_{3(0)} \cdot F_{4(I)} \cdot F_{5(0)} \cdot F_{6(I)} \cdot F_{7(0)} \cdot F_{8(I)}\right)\right)\end{array}$ & $Z$ \\
\hline 2 & $\Delta K_{e g}\left(F_{2}\right)=\left(\Delta F_{2} / 8\right)$ & $\begin{array}{l}2\left(\left(F_{1(0)} \cdot F_{3(I)} \cdot F_{4(0)} \cdot F_{5(I)} \cdot F_{6(0)} \cdot F_{7(I)} \cdot F_{8(0)}\right)+\right. \\
\left.+\left(F_{1(I)} \cdot F_{3(0)} \cdot F_{4(I)} \cdot F_{5(0)} \cdot F_{6(I)} \cdot F_{7(0)} \cdot F_{8(I)}\right)\right)\end{array}$ & $Z$ \\
\hline 3 & $\Delta K_{e g}\left(F_{3}\right)=\left(\Delta F_{3} / 8\right)$ & $\begin{array}{l}2\left(\left(F_{1(0)} \cdot F_{2(I)} \cdot F_{4(0)} \cdot F_{5(I)} \cdot F_{6(0)} \cdot F_{7(I)} \cdot F_{8(0)}\right)+\right. \\
\left.+\left(F_{1(I)} \cdot F_{2(0)} \cdot F_{4(I)} \cdot F_{5(0)} \cdot F_{6(I)} \cdot F_{7(0)} \cdot F_{8(I)}\right)\right)\end{array}$ & $Z$ \\
\hline 4 & $\Delta K_{e g}\left(F_{4}\right)=\left(\Delta F_{4} / 8\right)$ & $\begin{array}{l}2\left(\left(F_{1(0)} \cdot F_{2(I)} \cdot F_{3(0)} \cdot F_{5(I)} \cdot F_{6(0)} \cdot F_{7(I)} \cdot F_{8(0)}\right)+\right. \\
\left.+\left(F_{1(I)} \cdot F_{2(0)} \cdot F_{3(I)} \cdot F_{5(0)} \cdot F_{6(I)} \cdot F_{7(0)} \cdot F_{8(I)}\right)\right)\end{array}$ & $Z$ \\
\hline 5 & $\Delta K_{e g}\left(F_{5}\right)=\left(\Delta F_{5} / 8\right)$ & $\begin{array}{l}2\left(\left(F_{1(0)} \cdot F_{2(I)} \cdot F_{3(0)} \cdot F_{4(I)} \cdot F_{6(0)} \cdot F_{7(I)} \cdot F_{8(0)}\right)+\right. \\
\left.+\left(F_{1(I)} \cdot F_{2(0)} \cdot F_{3(I)} \cdot F_{4(0)} \cdot F_{6(I)} \cdot F_{7(0)} \cdot F_{8(I)}\right)\right)\end{array}$ & $Z$ \\
\hline 6 & $\Delta K_{e g}\left(F_{6}\right)=\left(\Delta F_{6} / 8\right)$ & $\begin{array}{l}2\left(\left(F_{1(0)} \cdot F_{2(I)} \cdot F_{3(0)} \cdot F_{4(I)} \cdot F_{5(0)} \cdot F_{7(I)} \cdot F_{8(0)}\right)+\right. \\
\left.+\left(F_{1(I)} \cdot F_{2(0)} \cdot F_{3(I)} \cdot F_{4(0)} \cdot F_{5(I)} \cdot F_{7(0)} \cdot F_{8(I)}\right)\right)\end{array}$ & $Z$ \\
\hline 7 & $\Delta K_{e g}\left(F_{7}\right)=\left(\Delta F_{7} / 8\right)$ & $\begin{array}{l}2\left(\left(F_{1(0)} \cdot F_{2(I)} \cdot F_{3(0)} \cdot F_{4(I)} \cdot F_{5(0)} \cdot F_{6(I)} \cdot F_{8(0)}\right)+\right. \\
\left.+\left(F_{1(I)} \cdot F_{2(0)} \cdot F_{3(I)} \cdot F_{4(0)} \cdot F_{5(I)} \cdot F_{6(0)} \cdot F_{8(I)}\right)\right)\end{array}$ & $Z$ \\
\hline 8 & $\Delta K_{e g}\left(F_{8}\right)=\left(\Delta F_{8} / 8\right)$ & $\begin{array}{l}2\left(\left(F_{1(0)} \cdot F_{2(I)} \cdot F_{3(0)} \cdot F_{4(I)} \cdot F_{5(0)} \cdot F_{6(I)} \cdot F_{7(0)}\right)+\right. \\
\left.+\left(F_{1(I)} \cdot F_{2(0)} \cdot F_{3(I)} \cdot F_{4(0)} \cdot F_{5(I)} \cdot F_{6(0)} \cdot F_{7(I)}\right)\right)\end{array}$ & $Z$ \\
\hline
\end{tabular}

Та блица 4

Результат по предложенному интегральному методу

\begin{tabular}{|c|c|c|c|c|}
\hline \multirow{2}{*}{$\begin{array}{c}\text { Номер } \\
\text { фактора }\end{array}$} & \multicolumn{5}{|c|}{ Части формулы } \\
\cline { 2 - 5 } & $\Delta F_{n} / n$ & $F O_{n}($ основная часть) & $Z$ & Итоговый результат \\
\hline 1 & $\Delta K_{e g}\left(F_{1}\right)=-0,000983146$ & 1,032064989 & 0,007991382 & 0,006976712 \\
\hline 2 & $\Delta K_{e g}\left(F_{2}\right)=-0,003968254$ & 1,229751152 & 0,007991382 & 0,003111418 \\
\hline 3 & $\Delta K_{e g}\left(F_{3}\right)=0,031250000$ & 0,172565587 & 0,007991382 & 0,013384057 \\
\hline 4 & $\Delta K_{e g}\left(F_{4}\right)=0,005998452$ & 1,045255986 & 0,007991382 & 0,014261300 \\
\hline 5 & $\Delta K_{e g}\left(F_{5}\right)=0,002083333$ & 2,315490452 & 0,007991382 & 0,012815321 \\
\hline 6 & $\Delta K_{e g}\left(F_{6}\right)=-0,037092732$ & 0,270203890 & 0,007991382 & $-0,000778393$ \\
\hline 7 & $\Delta K_{e g}\left(F_{7}\right)=0,002529839$ & 1,979232047 & 0,007991382 & 0,012998521 \\
\hline 8 & $\Delta K_{e g}\left(F_{8}\right)=0,030829832$ & 0,948421697 & 0,007991382 & 0,037231064 \\
\hline \multirow{2}{*}{ Всего } & $\left(\Delta F_{n} / n\right) F O_{n}$ & 0,063931060 & $\mathbf{0 , 1 0 0 0 0 0 0 0 0}$ \\
\cline { 2 - 3 } & 0,036068940 & & \\
\hline
\end{tabular}

Важнейший критерий деловой активности и надежности коммерческих организаций благоприятное финансово-экономическое состояние, определяющее его конкурентоспособность и потенциал в эффективной реализации экономических интересов [14]. Наращивание собственного капитала и обеспечение устойчивого положения на рынке является одной из основных задач финансовой деятельности предприятия [15]. Для это- 
го необходимо постоянно поддерживать платежеспособность и рентабельность предприятия, а также оптимальную структуру актива и пассива баланса.

Анализ предложенной ресурсноуправленческой модели может быть использован как:

- инструмент учета, анализа и планирования;

- индикатор научно-технического состояния хозяйственного субъекта и изучаемой совокупности;

- критерий сравнительного оценивания функционирования деятельности коммерческих организаций;

- показатель эффективности принятых ранее решений и полноты их реализации.

«Экономическая устойчивость каждого отдельного предприятия позволяет всей хозяйственной системе страны не только сохранить потенциал, но и обеспечить ее качественный подъем и выход на международные рынки с новыми конкурентоспособными товарами» [16, с. 55].

Изучение экономической действительности невозможно без аналитического подхода. Предложенный детерминированный фактор- ный анализ направлен на решение задачи поиска величин влияния изменения факторов, на изменение определяемого ими результирующего показателя, что определяет прикладное значение результатов исследований, направленных на качественное улучшение методологии данного вида анализа.

Предложенный интегральный метод направлен на качественное улучшение методологии данного вида анализа и расчет влияния любого количества факторов, используемых в исходной модели для анализа.

Обеспечение эффективного функционирования организаций требует экономически грамотного управления их деятельностью, которое во многом определяется умением ее анализировать. C помощью анализа изучаются тенденции развития, глубоко и системно исследуются факторы изменения результатов деятельности, обосновываются планы и управленческие решения, осуществляется контроль за их выполнением, выявляются резервы повышения эффективности производства, оцениваются результаты деятельности предприятия, вырабатывается экономическая стратегия его развития.

\section{СПИСОК ЛИТЕРАТУРЫ}

1. Чупров С.В. Управление устойчивостью производственных систем в условиях инновационной модернизации: дис. ... Д-ра экон. наук. Иркутск, 2008. 352 c.

2. Блюмин С.Л., Суханов В.Ф., Чеботарев С.В. Экономический факторный анализ: моногр. Липецк: ЛЭГИ, 2004. 148 с.

3. Экономический анализ / под ред. О.Н. Соколовой. М.: Инфра-М, 2011. 320 с.

4. Экономический анализ / под. ред. Л.Т. Гиляровской. М.: Юнити-Дана, 2001. 527 с.

5. Васильева Л.С., Петровская М.В. Финансовый анализ: учебник. 2-е изд. М.: КНОРУС, 2007. 816 с.

6. Чеботарев С.В. Теория и практика статического и динамического экономического факторного анализа // Системы управления и информационные технологии: межвуз. сб. науч. тр. Воронеж: Центр.-Черноз. кн. изд-во, 2001. С. 68-73.

7. Черкасов В.Е. Финансовый анализ в коммерческом банке. М.: Инфра-М, 1995. 272 с.

8. Черкасова И.О. Анализ хозяйственной деятельности: учеб. пособие. СПб.: Нева, 2009. 192 с.

9. Чечевицына Л.Н., Чуев И.Н. Анализ финансово-хозяйственной деятельности: учеб. пособие. 3-е изд. Ростов н/Дону: Феникс, 2006. 384 с.
10. Шеремет А.Д. Комплексный анализ хозяйственной деятельности: учебник. М.: Инфра-М, 2011. 415 c.

11. Ильенкова С.Д., Ильнкова Н.Д., Орехов С.А. и др. Экономико-статистический анализ: учеб. пособие для вузов / под ред. проф. С.Д. Ильенковой. М.: Юнити-Дана, 2002. 215 с.

12. Экономический анализ: ситуации, тесты, задачи, выбор оптимальных решений, финансовое прогнозирование: учеб. пособие / под ред. М.И. Баканова, А.Д. Шеремета. М.: Финансы и статистика, 2000. $656 \mathrm{c}$

13. Filatov E.A., Ryabchenkova A.V. Application of integral method in terms of 7-factor multiplicative model // Life Science Journal (USA), 2014, no. $11(9 \mathrm{~s})$, pp. $227-230$.

14. Feldstein M.A. Inflation, Tax Rules, and Investment: Some Econometric Evidence // Econometrica 50, 1982, no. 4 (July, 1982), pp. 825-862.

15. Doms M.E. Estimating Capital Efficiency Schedules Within Production Functions // Economic Inquiry 34, 1996, no. 1 (January, 1996), pp. 78-92.

16. Лайберт Т.Б. Экономическая устойчивость: сущность, содержание и факторы: учеб. пособие. Уфа: УГНТУ, 2008. 230 с. 


\section{REFERENCES}

1. Chuprov S.V. Upravlenie ustoichivostiu proizvodstvennykh sistem $\mathrm{v}$ usloviiakh innovatsionnoi modernizatsii: dis. ... d-ra ekon. nauk. Irkutsk, 2008. 352 s. (rus)

2. Bliumin S.L., Sukhanov V.F., Chebotarev S.V. Ekonomicheskii faktornyi analiz: monogr. Lipetsk: LEGI, 2004. 148 s. (rus)

3. Ekonomicheskii analiz. Pod red. O.N. Sokolovoi M.: Infra-M, 2011. 320 s. (rus)

4. Ekonomicheskii analiz. Pod. red. L.T. Giliarovskoi. M.: Iuniti-Dana, 2001. 527 s. (rus)

5. Vasil'eva L.S., Petrovskaia M.V. Finansovyi analiz: uchebnik. 2-e izd. M.: KNORUS, 2007. 816 s. (rus)

6. Chebotarev S.V. Teoriia i praktika staticheskogo i dinamicheskogo ekonomicheskogo faktornogo analiza. Sistemy upravleniia $i$ informatsionnye tekhnologii: mezhvuz. sb. nauch. tr. Voronezh: Tsentral'no-Cher. kn. izd-vo, 2001. S. 68-73. (rus)

7. Cherkasov V.E. Finansovyi analiz v kommercheskom banke. M.: Infra-M, 1995. 272 s. (rus)

8. Cherkasova I.O. Analiz khoziaistvennoi deiatel'nosti: ucheb. posobie. SPb.: Neva, 2009. 192 s. (rus)

9. Chechevitsyna L.N., Chuev I.N. Analiz finansovokhoziaistvennoi deiatel'nosti: ucheb. posobie. 3-e izd.
Rostov n/Donu: Feniks, 2006. 384 s. (rus)

10. Sheremet A.D. Kompleksnyi analiz khoziaistvennoi deiatel'nosti: uchebnik. M.: Infra-M, 2011. 415 s. (rus)

11. Il'enkova S.D., Il'nkova N.D., Orekhov S.A. i dr. Ekonomiko-statisticheskii analiz: ucheb. posobie dlia vuzov. Pod red. prof. S.D. Il'enkovoi. M.: Iuniti-Dana, 2002. 215 s. (rus)

12. Ekonomicheskii analiz: situatsii, testy, zadachi, vybor optimal'nykh reshenii, finansovoe prognozirovanie: ucheb. posobie. Pod red. M.I. Bakanova, A.D. Sheremeta. M.: Finansy i statistika, 2000. 656 s. (rus)

13. Filatov E.A., Ryabchenkova A.V. Application of integral method in terms of 7-factor multiplicative model. Life Science Journal (USA), 2014, no. 11(9s), pp. 227-230.

14. Feldstein M.A. Inflation, Tax Rules, and Investment: Some Econometric Evidence. Econometrica 50, 1982, no. 4 (July, 1982), pp. 825-862.

15. Doms M.E. Estimating Capital Efficiency Schedules Within Production Functions. Economic Inquiry 34, 1996, no. 1 (January, 1996), pp. 78-92.

16. Laibert T.B. Ekonomicheskaia ustoichivost': sushchnost', soderzhanie i faktory: ucheb. posobie. Ufa: UGNTU, 2008. 230 s. (rus)

ФИЛАТОВ Евгений Александрович - доцент Иркутского национального исследовательского технического университета, кандидат экономических наук.

664074, ул. Лермонтова, д. 83, г. Иркутск. E-mail: johnru3000@rambler.ru

FILATOV Evgenii A. - Irkutsk National Research Technical University.

664074. Lermontov str. 83. Irkutsk. Russia. E-mail: johnru3000@rambler.ru 